\title{
Hepatitis B virus strains of subgenotype A2 with an identical sequence spreading rapidly from the capital region to all over Japan in patients with acute hepatitis B
}

Yoko Tamada, ${ }^{1,2}$ Hiroshi Yatsuhashi, ${ }^{1,2}$ Naohiko Masaki, ${ }^{3}$ Makoto Nakamuta, ${ }^{4}$ Eiji Mita, ${ }^{5}$ Tatsuji Komatsu, ${ }^{6}$ Yukio Watanabe, ${ }^{7}$ Toyokichi Muro, ${ }^{8}$ Masaaki Shimada, ${ }^{9}$ Taizo Hijioka, ${ }^{10}$ Takeaki Satoh, ${ }^{11}$ Yutaka Mano, ${ }^{12}$ Toshiki Komeda, ${ }^{13}$ Masahiko Takahashi, ${ }^{14}$ Hiroshi Kohno, ${ }^{15}$ Hajime Ota, ${ }^{16}$ Shigeki Hayashi, ${ }^{17}$ Yuzo Miyakawa, ${ }^{18}$ Seigo Abiru, ${ }^{1,2}$ Hiromi Ishibashi ${ }^{1,2}$

For numbered affiliations see end of article.

\section{Correspondence to}

Professor Hiroshi Yatsuhashi, Clinical Research Center, NHO National Nagasaki Medical Center and Department of Hepatology, Nagasaki University Graduate School of Biomedical Sciences, Address: 2-1001-1 Kubara, Omura, Nagasaki 856-8562, Japan; yatsuhashi@nmc.hosp.go.jp

Revised 3 October 2011 Accepted 5 October 2011

\section{ABSTRACT}

Objective To examine recent trends of acute infection with hepatitis $B$ virus (HBV) in Japan by nationwide surveillance and phylogenetic analyses.

Methods During 1991 through 2009, a sentinel surveillance was conducted in 28 national hospitals in a prospective cohort study. Genotypes of HBV were determined in 547 patients with acute hepatitis B. Nucleotide sequences in the preS1/S2/S gene of genotype $A$ and $B$ isolates were determined for phylogenetic analyses.

Results HBV genotype A was detected in 137 (25\% (accompanied by genotype $\mathrm{G}$ in one)) patients, $\mathrm{B}$ in 48 $(9 \%), C$ in $359(66 \%)$, and other genotypes in the remaining three $(0.5 \%)$. HBV persisted in five with genotype $A$ including the one accompanied by genotype $\mathrm{G}$; another was co-infected with HIV type 1. The genotype was A in $4.8 \%$ of patients during 1991-1996, $29.3 \%$ during $1997-2002$, and $50.0 \%$ during

$2003-2008$ in the capital region, as against 6.5\%, 8.5\% and $33.1 \%$, respectively, in other regions. Of the 114 genotype $A$ isolates, $13(11.4 \%)$ were subgenotype $A 1$, and $101(88.6 \%)$ were $A 2$, whereas of the 43 genotype $B$ isolates, 10 (23.3\%) were subgenotype B1, 28 (65.1\%) were $\mathrm{B} 2$, two $(4.7 \%)$ were $\mathrm{B} 3$, and three $(7.0 \%)$ were B4. Sequences of $65(64 \%)$ isolates of $A 2$ were identical, as were three $(23 \%)$ of $A 1$, and five $(18 \%)$ of $B 2$, but none of the B1, B3 and B4 isolates shared a sequence. Conclusions Acute infection with HBV of genotype $A$, subgenotype A2 in particular, appear to be increasing, mainly through sexual contact, and spreading from the capital region to other regions in Japan nationwide. Infection persisted in $4 \%$ of the patients with genotype $A$, and HBV strains with an identical sequence prevailed in subgenotype A2 infections. This study indicates the need for universal vaccination of young people to prevent increases in HBV infection in Japan.

Hepatitis B virus (HBV) has been classified into 10 genotypes, designated $\mathrm{A}-\mathrm{J}$, based on a $>8 \%$ divergence in the full-genome sequence. ${ }^{1-7}$ Different genotypes are associated with distinct clinical manifestations, such as severity and progression of

\section{Significance of this study}

What is already known about this subject?

- In Japan, a national prevention programme was started in 1986 with selective vaccination of babies born to mothers who carry hepatitis $B$ virus (HBV). Since then, the prevalence of hepatitis B surface antigen among younger generations has decreased sharply.

- However, retrospective studies indicate that the frequency of HBV genotype $A$ is increasing among patients with acute hepatitis $B(A H B)$ within the capital region of Japan.

- Infection with genotype A more often persists than infection with other genotypes.

- Because there is no reliable and comprehensive surveillance system for AHB in Japan, the incidence of $A H B$ and factors responsible for changes over many years are not known.

What are the new findings?

- This is a prospective cohort study for surveillance of AHB throughout Japan in a national research programme.

- The incidence of AHB in Japan has not decreased, because genotype A infections have increased over time.

- Genotype A infections started to increase in the capital region of Japan, and then spread to other regions $5-6$ years later.

- About $90 \%$ of genotype A found in AHB patients in Japan is subgenotype A2.

- Subgenotype A2 isolates from patients with AHB tend to preserve sequence identity over time, indicating that particular subgenotype A2 strains have been transmitted without undergoing mutations.

liver disease, as well as response to antiviral treatments. $^{8-10}$ Some genotypes are subclassified: genotype $\mathrm{A}$ into at least two subgenotypes, A1 (Asian/African type) and A2 (European type) ${ }^{11-13}$; 


\section{Significance of this study}

How might it impact on clinical practice in the foreseeable future?

- It needs to be noted that subgenotype A2 infections are spreading among sexually active generations in Japan.

- Although selective vaccination has prevented mother-to-baby transmission of HBV since 1986, it does not contain sporadic infections in Japan.

- Herd vaccination of younger generations needs to be considered in Japan.

B into B1 (Japanese type) and B2 (Asian type) ${ }^{14}{ }^{15}$; and C into C1 (Southeast-Asian type) and C2 (East-Asian type). ${ }^{16}$ Subgenotypes also influence the replication of $\mathrm{HBV}$ and clinical manifestation. ${ }^{15} 1718$

According to a report from Japan in $2001,{ }^{19}$ genotype $\mathrm{C}$ was the most prevalent $(84.7 \%)$, followed by genotype B $(12.2 \%)$ and A $(1.7 \%)$, among patients with chronic hepatitis B. In 2002, genotype $A$ became the most prevalent in patients with acute hepatitis B (AHB) around Tokyo, the capital region of Japan. ${ }^{20} 21$ Several reports have shown that infection with HBV genotype A is associated with particular sexual behaviours, such as homosexual activity and promiscuous sexual contacts, and tends to persist longer than that with HBV genotype C. ${ }^{22} 23$ These reports have raised concerns about the horizontal HBV infection in adults, which, in general, is considered to resolve spontaneously. However, adult-acquired HBV infection may result in chronic HBV infection in some instances.

Information on changes in genotype distribution over time, as well as genotype-specific clinical manifestations, may help in planning preventive measures and antiviral therapy strategies. Therefore it is important to examine how genotype A infection has spread in Japan, and what clinical and virological characteristics it possesses.

We have been conducting a nationwide, sentinel surveillance on acute viral hepatitis for more than 30 years. As part of this surveillance, a prospective cohort study has been conducted on 547 patients with AHB in 28 medical centres over the 19 years from 1991 to 2009. Geographical and longitudinal distributions of HBV genotypes/subgenotypes were surveyed, and their influence on clinical outcome was evaluated.

\section{PATIENTS AND METHODS}

\section{Patients}

A total of 681 patients with sporadic AHB were enrolled consecutively in a survey carried out by the Japan National Hospital Acute Hepatitis Study Group (JNHAHSG). They were admitted to 28 national hospitals from January 1991 to the end of December 2009. They were grouped geographically into two areas: the capital region (Gunma, Saitama, Tokyo and Kanagawa) and other regions (figure 1). Patients were also longitudinally categorised into three periods: 1st (1991-1996), 2nd (1997-2002) and 3rd (2003-2008). In addition, the year 2009 provided the most recent data. Of the 681 patients, $547(80.3 \%)$ entered the study, for whom serum samples were available on admission and had been stored at $-20^{\circ} \mathrm{C}$.

The diagnosis of AHB was based on the following criteria: (1) acute onset of liver injury without a history of liver dysfunction; (2) detection of hepatitis B surface antigen (HBsAg) in the

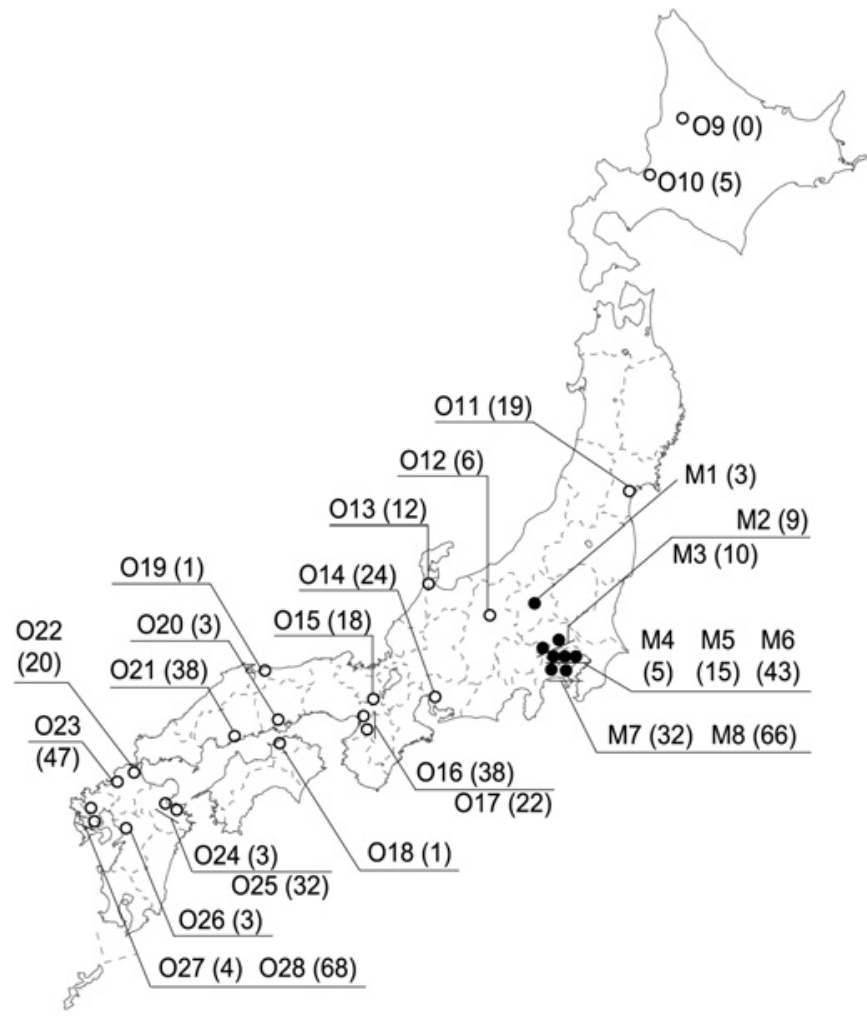

Figure 1 Locations of participating hospitals in Japan. Hospitals in the capital region (M1-M8) are indicated by eight closed circles, and those in other regions $(09-028)$ by 20 open circles. Numbers in parentheses indicate the total number of enrolled subjects for each site. The hospitals are: M1, Nishigunma Hospital, Gunma; M2, Nishisaitama-Chuo Hospital, Saitama; M3, National Disaster Medical Center, Tokyo; M4, Tokyo Hospital, Tokyo; M5, Tokyo Medical Center, Tokyo; M6, National Center for Global Health and Medicine, Tokyo; M7, Sagamihara Hospital, Kanagawa; M8, Yokohama Medical Center, Kanagawa; 09, Asahikawa Medical Center, Hokkaido; 010, Hokkaido Medical Center, Hokkaido; 011, Sendai Medical Center, Miyagi; 012, Matsumoto Medical Center, Nagano; 013, Kanazawa Medical Center, Ishikawa; 014, Nagoya Medical Center, Aichi; 015, Kyoto Medical Center, Kyoto; 016, Osaka National Hospital, Osaka; 017, Osaka-Minami Medical Center, Osaka; 018, Zentsuji Hospital, Kagawa; 019, Yonago Medical Center, Tottori; 020, Okayama Medical Center, Okayama; 021, Kure Medical Center and Chugoku Cancer Center, Hiroshima; 022, Kokura Medical Center, Fukuoka; 023, Kyushu Medical Center, Fukuoka; 024, Beppu Medical Center, Oita; 025, Oita Medical Center, Oita; 026, Kumamoto Medical Center, Kumamoto; 027, Ureshino Medical Center, Saga; and 028, Nagasaki Medical Center, Nagasaki.

serum; (3) positivity for IgM antibody to HBV-core antigen (IgM anti-HBc) in high titres (detectable in sera diluted 10-fold); and (4) absence of past or family history of chronic HBV infection. Severe acute hepatitis $(\mathrm{SAH})$ was defined as prothrombin time (PT) $\leq 40 \%$ and hepatic encephalopathy of grade $\leq$ I. Fulminant hepatitis $(\mathrm{FH})$ was diagnosed from PT $\leq 40 \%$ and hepatic encephalopathy of grade $\geq$ II. Patients in whom HBsAg remained in the serum for $>6$ months after onset were considered to have acquired chronic HBV infection. The following information was collected from each patient: year and age at onset, gender, residential area, $\mathrm{HBsAg}, \operatorname{IgM}$ anti-HBc, alanine aminotransferase, total bilirubin, PT, severity of liver disease, mortality, routes of transmission, sexual behaviours, travelling abroad in recent past, HBV genotype, mutations in precore (PreC) and core promoter (CP) regions, and RNA of hepatitis D virus. Antibody to HIV type 1 (anti-HIV) was 
determined in patients who were at high risk and gave consent to testing.

Informed consent was obtained from each patient. The study protocol conforms to the ethical guidelines of the 1975 Declaration of Helsinki and the Ministry of Education, Culture, Sports Science and Technology of Japan, and was approved by the ethics committee of each institution.

\section{Extraction of HBV DNA}

HBV DNA was extracted from serum $(100 \mu \mathrm{l})$ by the SMITEST EX-R\&D Nucleic Acid Extraction Kit (MBL Co, Nagoya, Japan) and used for genotyping/subgenotyping and detecting mutations in PreC and CP regions.

\section{HBV genotypes}

Genotypes were determined in Nagasaki Medical Center with the SMITEST HBV Genotyping Kit (MBL) by hybridisation with type-specific probes immobilised on a solid-phase support. $^{24}$

\section{Determination of HBV subgenotypes}

For subgenotyping, HBV DNA was amplified by PCR with TaKaRa Ex Taq (Takara Bio, Shiga, Japan). PCR was performed with appropriate nested primers to amplify a $\sim 1.2 \mathrm{~kb}$ sequence in the preS1/S2/S gene (nucleotides 2854-835 in the reference isolate (AB116077)). PCR products were purified, subjected to cycle sequencing reaction with the BigDye Terminator v1.1 (Applied Biosystems, Tokyo, Japan), and applied to the DNA sequencer (3100-Avant; Applied Biosystems).

\section{Mutations in the PreC and CP regions}

The A1896 mutation in the PreC region was detected by the enzyme-linked minisequence assay (SMITEST HBV PreC ELMA; Roche Diagnostics, Tokyo, Japan), and mutations in the CP region for T1762/A1764 by the enzyme-linked specific probe assay (SMITEST HBV Core Promoter Mutation Detection Kit; Roche Diagnostics). The results were recorded as 'wild-type' and 'mutant types' dominantly expressed by HBV isolates. ${ }^{25}$

\section{Phylogenetic analyses}

Nucleotide sequences were aligned, and phylogenetic trees were constructed by the CLUSTAL W program v1.83 (DDBJ homepage: http://clustalw.ddbj.nig.ac.jp/top-j.html). The statistical validity was assessed by bootstrap resampling with 1000 replicates. Reference HBV strains were retrieved from the GenBank database.

\section{Statistical analysis}

Results were expressed as percentage or mean \pm SD. Statistical differences were evaluated by $\chi^{2}$ and Fisher exact tests for categorical variables, and analysis of variance and Scheffe's test for quantitative variables, using the SPSS software. The 95\% CI, for the difference in means, was calculated in analyses for quantitative variables. $\mathrm{p}<0.05$ was considered significant.

\section{RESULTS}

\section{Distribution of HV genotypes}

HBV genotypes were determined in the 547 patients with AHB. The genotype was A in 137 (25.0\%) patients (accompanied by G in one $(0.2 \%)), \mathrm{B}$ in $48(8.8 \%), \mathrm{C}$ in $359(65.6 \%), \mathrm{D}$ in one $(0.2 \%)$, $\mathrm{E}$ in one $(0.2 \%)$, and $\mathrm{H}$ in one $(0.2 \%)$. Because HBV genotype $\mathrm{G}$ is a defective virus and cannot replicate by itself, ${ }^{26} 27$ the single patient with mixed genotypes A and G was included in the 137 patients with genotype $\mathrm{A}$ in further analyses. RNA of hepatitis
$\mathrm{D}$ virus was detected in three of the $453(0.7 \%)$ patients. AntiHIV was examined in patients at high risk of infection and detected in 14 of the 53 (26.4\%) who gave consent to testing.

\section{Demographic and clinical differences among patients infected with HBV of distinct genotypes}

Demographic and clinical characteristics of patients with different genotypes are compared in table 1. There was no difference in mean age among patients with genotypes A, B and $C$. The proportion of men was higher in patients with genotype A than B or C $(94.2 \%$ vs $79.2 \%, p<0.05$; or $56.0 \%, p<0.0001)$, and in those with genotype B than C $(79.2 \%$ vs $56.0 \%, p<0.05)$.

Maximum levels of total bilirubin were higher in patients with genotype A than C (9.6 \pm 7.6 vs $7.1 \pm 6.2 \mathrm{mg} / \mathrm{dl}, \mathrm{p}<0.05)$, with a difference of $2.5 \mathrm{mg} / \mathrm{dl}$ (95\% CI 0.93 to 4.08$)$, whereas the highest alanine aminotransferase activity and lowest PT values did not differ among patients with distinct genotypes.

$\mathrm{SAH}$ developed in four (2.9\%) patients with genotype A, four (8.3\%) with genotype B, and 26 (7.2\%) with genotype C. FH developed in one $(2.1 \%)$ patient with genotype B and eight $(2.2 \%)$ with genotype C; no patients with genotype A developed $\mathrm{FH}$. Eight (1.5\%) patients died, including one with genotype B and seven with genotype $C$. There were no significant differences among patients with different genotypes in the frequency of SAH or FH or mortality.

The outcome of AHB was traceable in 514 of the 547 (94.0\%) patients. Chronic infection with persistence of $\mathrm{HBsAg}$ for $>6$ months developed in five of the 123 (4.1\%) patients with genotype A (including the one accompanied by genotype $\mathrm{G}$ ), none of the $46(0 \%)$ with genotype $B$, and none of the $342(0 \%)$ with genotype $\mathrm{C}$; it was more common in patients with genotype A than $\mathrm{C}(\mathrm{p}<0.05)$. HBV infection persisted exclusively in the patients with genotype $A$, either alone (four patients) or together with genotype $G$ (one).

Among the five patients who acquired chronic HBV infection, four (three with genotype $\mathrm{A}$ and one with mixed genotypes $\mathrm{A}$ and $G$ ) were examined for anti-HIV, and one with genotype A was found to be positive. HBV infection persisted in three (including the one with anti-HIV) of the five patients for $>1$ year after the onset, and the remaining two (both without anti-HIV) cleared HBsAg from the serum after retaining it for $>6$ months.

Mutations in the PreC and/or CP region were detected in 3.7\% (4/109) of patients with genotype A, 15.4\% (6/39) of those with genotype $B$, and $25.5 \%(79 / 310)$ of those with genotype $C$. They were significantly less common in patients with genotype $A$ than $B$ or $C$ (A vs $B, p<0.05$; A vs $C, p<0.0001$ ). The only patient with genotype $\mathrm{A}$ who had the PreC mutation was simultaneously infected with genotype G

Routes of transmission were identifiable in 275 of the 547 $(50 \%)$ patients, and the main route was heterosexual contacts; those in the remaining patients could not be disclosed. The frequency of heterosexual activity did not differ among patients with distinct genotypes. However, homosexual activity was more common in patients with genotype $\mathrm{A}$ than $\mathrm{B}$ or $\mathrm{C}(21.2 \%$, $0 \%$ and $0.8 \%$, respectively ( $A$ vs $B, p<0.001$; $A$ vs $C, p<0.0001$ )). Among the 32 homosexual men, HBV genotype A was detected in $29(91 \%)$. Consent to anti-HIV testing was given by 10 of the 29 patients, and four of these $(40 \%)$ were positive.

\section{Longitudinal changes in the distribution of genotypes}

Figure 2 illustrates changes in the distribution of HBV genotypes through three 6-year periods over 18 years (1991-2008). In addition, data from 2009 are shown. HBV genotype A accounted 
Table 1 Demographic and clinical characteristics of patients with acute hepatitis who were infected with HBV of different genotypes (1991-2009)

\begin{tabular}{|c|c|c|c|c|c|}
\hline \multirow[b]{2}{*}{ Feature } & \multirow[b]{2}{*}{ Total $(n=547)$} & \multicolumn{4}{|l|}{ HBV genotypes } \\
\hline & & $A(n=137)+(25.0 \%)$ & $B(n=48)(8.8 \%)$ & C $(n=359)(65.6 \%)$ & Others $(n=3) \neq(0.5 \%)$ \\
\hline Age (years) & $35.6 \pm 14.8$ & $35.2 \pm 12.2$ & $39.6 \pm 15.6$ & $35.1 \pm 15.5$ & $49.7 \pm 13.6$ \\
\hline Male & $367(67.1 \%)$ & $129(94.2 \%) \Phi * \overbrace{}^{*} \dagger{ }^{* * *}$ & $38(79.2 \%) \dagger^{*}$ & $201(56.0 \%)$ & $3(100 \%)$ \\
\hline T-Bil $(\mathrm{mg} / \mathrm{dl}) \S$ & $7.8 \pm 6.7$ & $9.6 \pm 7.6 \dagger \dagger^{*}$ & $7.7 \pm 7.4$ & $7.1 \pm 6.2$ & $9.0 \pm 2.5$ \\
\hline PT $(\%) \S$ & $74.6 \pm 22.6$ & $75.2 \pm 15.9$ & $73.8 \pm 24.5$ & $74.7 \pm 24.5$ & $15.8 \neq \ddagger$ \\
\hline Severe hepatitis & $34(6.2 \%)$ & $4(2.9 \%)$ & $4(8.3 \%)$ & $26(7.2 \%)$ & $0(0.0 \%)$ \\
\hline HBsAg persisting $>6$ months & $5 / 514(1.0 \%)$ & $5 / 123(4.1 \%)+\dagger^{*}$ & $0 / 46(0.0 \%)$ & $0 / 342(0 \%)$ & $0 / 3(0.0 \%)$ \\
\hline \multicolumn{6}{|l|}{$\mathrm{PreC} / \mathrm{CP}$ mutations } \\
\hline PreC & $43 / 461(9.3 \%)$ & $1 / 109(0.9 \%) \Phi{ }^{*} \dagger \dagger *$ & $6 / 39(15.4 \%)$ & $34 / 310(11.0 \%)$ & $2 / 3(66.7 \%)$ \\
\hline $\mathrm{CP}$ & $69 / 461(15.0 \%)$ & $3 / 109(2.8 \%) \dagger \dagger * * *$ & $0 / 39(0.0 \%)+\dagger^{*}$ & $63 / 310(20.3 \%)$ & $3 / 3(100 \%)$ \\
\hline PreC and/or $\mathrm{CP}$ & $92 / 461(20.0 \%)$ & $4 / 109(3.7 \%) \uparrow{ }^{*} \dagger \dagger^{* * *}$ & $6 / 39(15.4 \%)$ & $79 / 310(25.5 \%)$ & $3 / 3(100 \%)$ \\
\hline Other & $10(1.8 \%)$ & $1(0.7 \%)$ & $1(2.1 \%)$ & $7(1.9 \%)$ & $1(33.3 \%)$ \\
\hline Undetermined & $272(49.7 \%)$ & $53(38.7 \%) \dagger \dagger^{*}$ & $20(41.7 \%)$ & $198(55.2 \%)$ & $1(33.3 \%)$ \\
\hline Anti-HIV & $14 / 53(26.4 \%)$ & $11 / 35(31.4 \%)$ & $0 / 3(0.0 \%)$ & $3 / 15(20.0 \%)$ & $0 / 0$ \\
\hline
\end{tabular}

Values are mean \pm SD or number (\%).

tOne patient with genotype A was simultaneously infected with genotype $\mathrm{G}$.

$\ddagger$ Each patient was infected with genotype $\mathrm{D}, \mathrm{E}$ or $\mathrm{H}$.

SHighest values during the clinical course are shown for ALT and T-Bil, and lowest values for PT.

Statistical analysis was performed to compare genotypes A, B and C.

qSignificantly different compared with genotype $B$.

$\dagger †$ Significantly different compared with genotype $\mathrm{C}$.

${ }^{*} \mathrm{p}<0.05,{ }^{* *} \mathrm{p}<0.001,{ }^{* * *} \mathrm{p}<0.0001$.

$\neq \neq$ Data from the patient with genotype $E$ only.

ALT, alanine aminotransferase; $\mathrm{CP}$, core promoter; $\mathrm{HBsAg}$, hepatitis B surface antigen; HBV, hepatitis B virus; PreC, precore; PT, prothrombin time; T-Bil, total bilirubin.

for $6 \%(9 / 150)$ in the 1 st period, $15.4 \%(19 / 123)$ in the $2 \mathrm{nd}$, and $39.4 \%(89 / 226)$ in the 3rd, with significant differences between 1 st and 2nd ( $<<0.05), 2$ nd and 3rd ( $<<0.0001)$, and 1st and 3rd $(p<0.0001)$. Conversely, AHB associated with genotype C decreased through three periods with significant differences, while AHB associated with genotype B did not change appreciably.

On the basis of these results, the yearly incidence in each of the three 6-year periods is calculated to be: 25.0 cases including 1.5 with genotype $\mathrm{A}$ in the 1 st period; 20.5 cases including 3.2 with genotype $A$ in the 2 nd; and 37.7 cases including 14.8 with genotype $\mathrm{A}$ in the 3rd. Hence, the incidence of AHB had not changed markedly over the 12 years from 1991 to 2002, but increased thereafter until 2008. Of the increment in the $3 \mathrm{rd}$ period of 17.2 (37.7 minus 20.5) cases, there were 11.6 (14.8 minus 3.2) with genotype A; they accounted for $67 \%(11.6 / 17.2)$ of the recent increase in AHB.

\section{Regional distributions and longitudinal changes in genotype A}

Among the 183 patients from the capital region, the genotype was $\mathrm{A}$ in $65(35.5 \%), \mathrm{B}$ in $22(12.0 \%), \mathrm{C}$ in $94(51.4 \%), \mathrm{E}$ in one $(0.5 \%)$, and $\mathrm{H}$ in one $(0.5 \%)$ (table 2$)$. Of the remaining 364 $(66.5 \%)$ patients from other regions, by contrast, the genotype was A in $72(19.8 \%), \mathrm{B}$ in $26(7.1 \%), \mathrm{C}$ in $265(72.8 \%)$, and $\mathrm{D}$ in one $(0.3 \%)$. Genotype A was significantly more common in the capital than in other regions ( $35.5 \%$ vs $19.8 \%, \mathrm{p}<0.0001)$. In the capital region, genotype A accounted for $4.8 \%(2 / 42)$ in the 1 st period, $29.3 \%(12 / 41)$ in the $2 \mathrm{nd}$, and $50.0 \%(42 / 84)$ in the $3 \mathrm{rd}$. There were significant differences between the 1st and 2 nd periods $(p<0.05)$, 2nd and 3rd $(p<0.05)$, and 1 st and 3rd $(p<0.0001)$. In other regions, by contrast, genotype $A$ accounted for $6.5 \%(7 / 108)$ in the 1 st period, $8.5 \%(7 / 182)$ in the $2 \mathrm{nd}$, and
$33.1 \%(47 / 142)$ in the 3rd. For the first time in other regions, genotype $\mathrm{A}$ increased in the 3rd period, in comparison with the 1 st and 2 nd (1st vs 3rd, $\mathrm{p}<0.0001 ; 2$ nd vs 3rd, $\mathrm{p}<0.0001$ ).

\section{Subgenotypes of genotype A}

Of the 137 genotype $\mathrm{A}$ isolates, amplification and sequencing of HBV DNA were feasible in 114 (83.2\%); the isolate from the single patient with genotypes $A$ and $G$ was excluded. A phylogenetic tree was constructed, on the entire preS1/S2/S genes of $\sim 1.2 \mathrm{~kb}$, for these 114 isolates along with 34 genotype A isolates retrieved from the database (figure 3 ).

Of the 114 isolates in this study, 101 (88.6\%) were subgenotype $\mathrm{A} 2$, and the remaining 13 (11.4\%) were subgenotype A1. In a pair-wise comparison, the sequence divergence among the 101 subgenotype A2 isolates was $0-1.3 \%$, and that among the 13 subgenotype A1 isolates spanned $0 \%$ to $2.3 \%$. The sequence divergence between subgenotype $\mathrm{A} 2$ and $\mathrm{A} 1$ isolates ranged from $2.6 \%$ to $4.7 \%$.

A sequence of 1203 nucleotides was possessed in common by three of the $101(3 \%)$ isolates of subgenotype A2. For convenience, the group comprising these three isolates was labelled 'identical group I'. Likewise, an additional six 'identical groups' were found, and numbered from 'II' to 'VII'. They comprised 35 $(35 \%)$, seven $(7 \%)$, two $(2 \%)$, three $(3 \%), 12(12 \%)$ and three $(3 \%)$ of the 101 isolates of subgenotype A2. In contrast, only one identical group, designated 'VIII', was constructed by three of the $13(23 \%)$ isolates of subgenotype A1.

Some isolates of subgenotype A1 and A2 were obtained from patients who had travelled to foreign countries in the recent past (5/13 (38.5\%) patients with A1 to Africa, Philippines, Myanmar and China; and 5/101 (5.0\%) patients with A2 to Europe, Thailand, Brazil and the USA). 
Figure 2 Distribution of hepatitis B virus (HBV) genotypes in three periods.

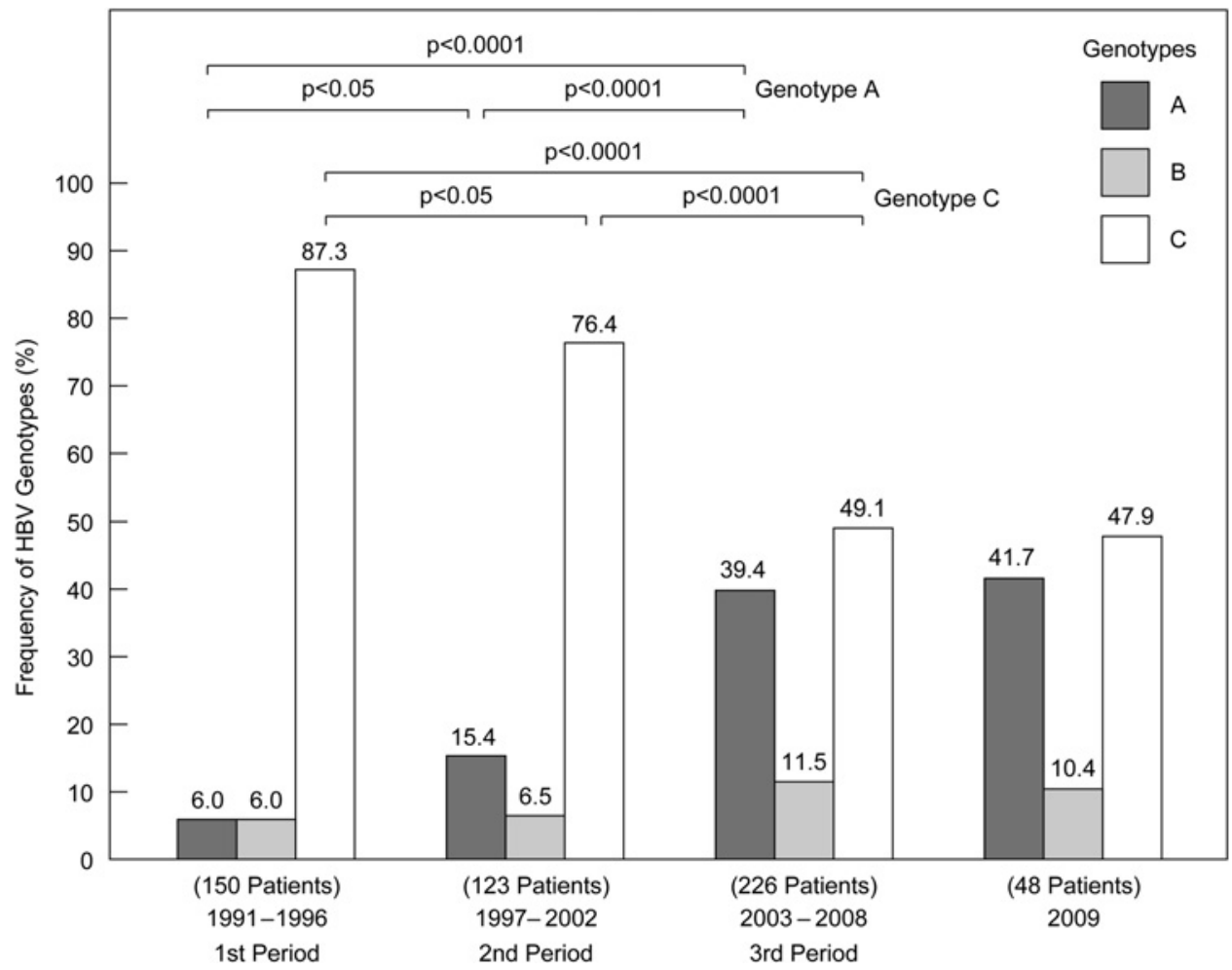

\section{Subgenotypes of genotype B}

Of the 48 isolates of genotype $B$, subgenotyping was feasible in $43(90.0 \%)$. A phylogenetic tree was constructed on preS1/S2/Sgene sequences from these 43 isolates, along with those from 25 isolates of genotype $B$ retrieved from the database (figure 4). Of the 43 isolates in this study, 10 (23.3\%) were subgenotype B1, 28 $(65.1 \%)$ were B2, two (4.7\%) were B3, and three (7.0\%) were B4. In a pair-wise comparison, the sequence divergence among 10 subgenotype B1 isolates ranged from $0.4 \%$ to $1.4 \%$, and that among 28, two and three isolates of subgenotypes B2, B3 and B4 spanned $0-1.7 \%, 0.5 \%$ and $0.6-0.8 \%$, respectively. The inter-subgenotype divergence among B1-B4 ranged from $0.6 \%$ to $4.4 \%$.

One 'identical group' made up of five isolates was detected among the 28 of subgenotype B2; it was named 'IX'. In contrast, no 'identical group' was found in 10, two or three isolates of subgenotype B1, B3 or B4.

Some isolates of subgenotypes B2, B3 and B4 were obtained from patients who had travelled to foreign countries in the recent past $(7 / 28(25.0 \%)$ patients with $\mathrm{B} 2$ to China and other countries; $1 / 2(50.0 \%)$ patients with B3 to a country unknown; and $1 / 3(33.3 \%)$ patients with B4 to Vietnam). However, none of the 10 subgenotype B1 isolates was associated with travel to foreign countries.

\section{Identical groups}

The proportion of isolates that shared a sequence in identical groups was higher for subgenotype A2 (64.4\%) than for A1, B1, $\mathrm{B} 2$, B3 or $\mathrm{B} 4(23.1 \%, 0 \%, 17.9 \%, 0 \%$ or $0 \%$, respectively (A2 vs $\mathrm{A} 1, \mathrm{p}<0.001$; $\mathrm{A} 2$ vs B1, $\mathrm{p}<0.0001$; $\mathrm{A} 2$ vs $\mathrm{B} 2, \mathrm{p}<0.0001)$ ).

Homosexual activity was more common in patients belonging to the seven identical groups than the non-identical group of subgenotype A2 (17/65 (26.2\%) vs 3/36 (8.3\%), p<0.05). Among the isolates in the seven identical groups of subgenotype A2, those in groups I, III and VII clustered locally during short periods of 2-7 years. In contrast, subgenotype A2 isolates in groups II and VI were scattered widely over longer periods of 11-16 years.

\section{DISCUSSION}

In Japan, as in most Asian countries, the persistent HBV carrier state had been established mainly through perinatal transmission from mother to baby and horizontal infection during infancy. In 1986, a national prevention programme was launched in Japan with selective vaccination of babies born to carrier mothers with hepatitis B e antigen (HBeAg). In 1995, this was extended to babies born to HBeAg-negative carrier mothers. As a result, the prevalence of HBsAg among younger people born since 1986 has decreased dramatically. ${ }^{28} 29$ However, there are an

Table 2 Changes in the distribution of genotype A compared between the capital region and other regions over three periods

\begin{tabular}{|c|c|c|c|c|c|}
\hline Area & $\mathbf{n}$ & $\begin{array}{l}\text { 1st Period } \\
(1991-1996)\end{array}$ & $\begin{array}{l}\text { 2nd Period } \\
(1997-2002)\end{array}$ & $\begin{array}{l}\text { 3rd Period } \\
(2003-2008)\end{array}$ & 2009 \\
\hline Capital region & $65 / 183(35.5 \%) \dagger^{* * *}$ & $2 / 42(4.8 \%) \ddagger^{*} \S^{* * *}$ & $12 / 41(29.3 \%) \dagger^{*} \S^{*}$ & $42 / 84(50.0 \%) \dagger^{*}$ & $9 / 16(56.3 \%)$ \\
\hline Other regions & $72 / 364$ (19.8\%) & $7 / 108(6.5 \%) \S^{* * *}$ & $7 / 82(8.5 \%) \S^{* * *}$ & $47 / 142(33.1 \%)$ & $11 / 32(34.4 \%)$ \\
\hline Total & $137 / 547(25.0 \%)$ & $9 / 150(6.0 \%) \ddagger^{*} \S^{* * *}$ & $19 / 123(15.4 \%) \S^{* * *}$ & $89 / 226(39.4 \%)$ & $20 / 48(41.7 \%)$ \\
\hline
\end{tabular}

Statistical analysis of the differences between the capital and other regions was performed, as well as through the 1st, 2nd and 3rd periods.

†Significantly different compared with other regions.

¥Significantly different compared with the 2nd period.

$\S$ Significantly different compared with the 3rd period.

${ }^{*} \mathrm{p}<0.05,{ }^{* *} \mathrm{p}<0.0001$. 
Figure 3 Phylogenetic analysis of genotype $A$ strains by the neighbourjoining method. Isolates obtained in this study are shown in bold with asterisks. Hospitals in the capital region are labelled M1-M8 and those in other regions 09-028 (corresponding to those in figure 1). Year of onset is indicated by the last two digits after the first hyphen. Numbers after the second hyphen represent the identification numbers of patients in each year (not always consecutive). Transmission routes are shown in lower-case letters in parentheses: $h$, homosexual; $s$, heterosexual; $\mathrm{m}$, medical procedure; 0 , others; and $\mathrm{u}$, undetermined. Isolates with identical sequences are bracketed in 'Identical groups I through VIII' on the tree. Each bracket is divided by areas and periods. Reference hepatitis $B$ virus (HBV) isolates, including 12 of subgenotype $A 1$ and 22 of subgenotype $A 2$, were obtained from the database and specified by their accession numbers, isolate names and countries of origin. Bootstrap values are indicated on major phylogenetic branches.

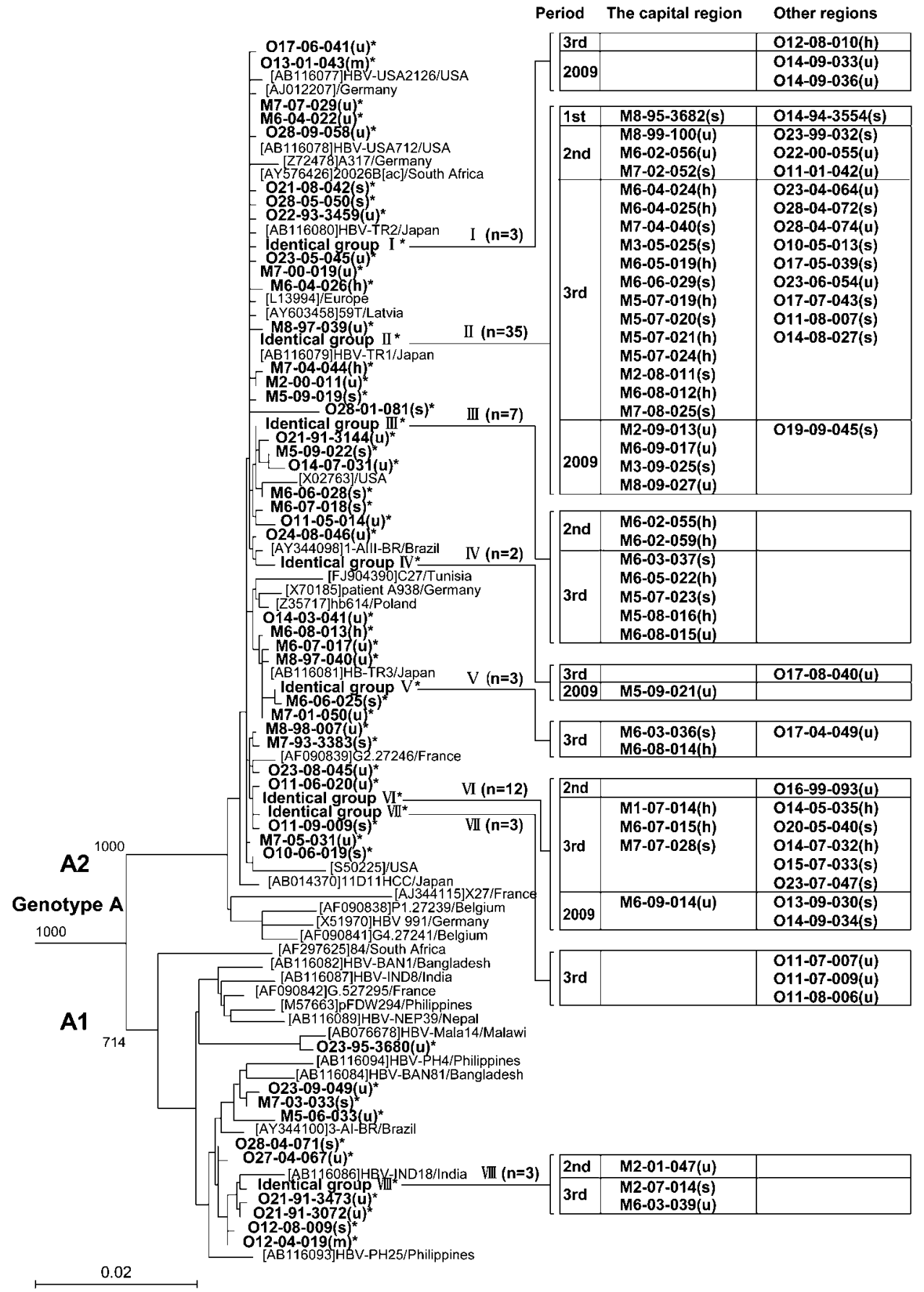

estimated one million HBV carriers in Japan at present. ${ }^{30}$ Furthermore, many Japanese remain at increased risk of horizontal infection with $\mathrm{HBV}$, because they have not received selective vaccination and therefore do not have the antibody to HBsAg. Because AHB is extremely under-reported and no national surveillance data are available in Japan, the incidence has not been determined accurately. In the USA, the incidence of AHB has decreased markedly since the adoption of a comprehensive immunisation strategy in 1991. ${ }^{31} 32$

In the present study over 1991-2009, we conducted a nationwide, sentinel surveillance on AHB in Japan. In the 547 patients recruited over 19 years, genotype $\mathrm{C}$ was the most prevalent $(65.6 \%)$, followed by genotype A $(25.0 \%)$ and genotype B (8.8\%). Demographic and clinical differences were observed among patients with genotypes A, B and C (table 1).
The proportion of men reached $94.2 \%$ for genotype A infection, higher than that for genotype B $(79.2 \%)$ or C $(56.0 \%)$ infection. In the analysis of the route of transmission, homosexual activity was reported by $21.2 \%$ of patients with genotype $A$; all were male. In general, sexual activity tends to be higher in men than women. The predominance of genotype $\mathrm{A}$ in men may be attributable to a high frequency of homosexual activity among men.

Although adult-acquired HBV infection persists at a high frequency of $\sim 10 \%$ in European countries and the USA, ${ }^{33}$ it rarely, if ever, becomes chronic in Japan. Recent studies suggest that the chance of a chronic outcome of AHB may differ by HBV genotype $^{21}{ }^{34}$; it is more common for genotype $\mathrm{A}$ than other genotypes. $^{22} 3536$ In the present study, HBV infection persisted in $4.1 \%$ of patients with genotype $\mathrm{A}$, in comparison with $0 \%$ of 
Figure 4 Phylogenetic analysis of genotype $B$ strains by the neighbourjoining method. Hepatitis B virus (HBV) isolates obtained in the present study are specified in the same manner as in figure 3 , and isolates with an identical sequence are bracketed in 'Identical group IX' on the tree. Of them, 10 reference isolates of subgenotype $\mathrm{B} 1$ and 13 , two and two of those of B2, B3 and $B 4$, respectively, were retrieved from the database; they are specified as in figure 3.

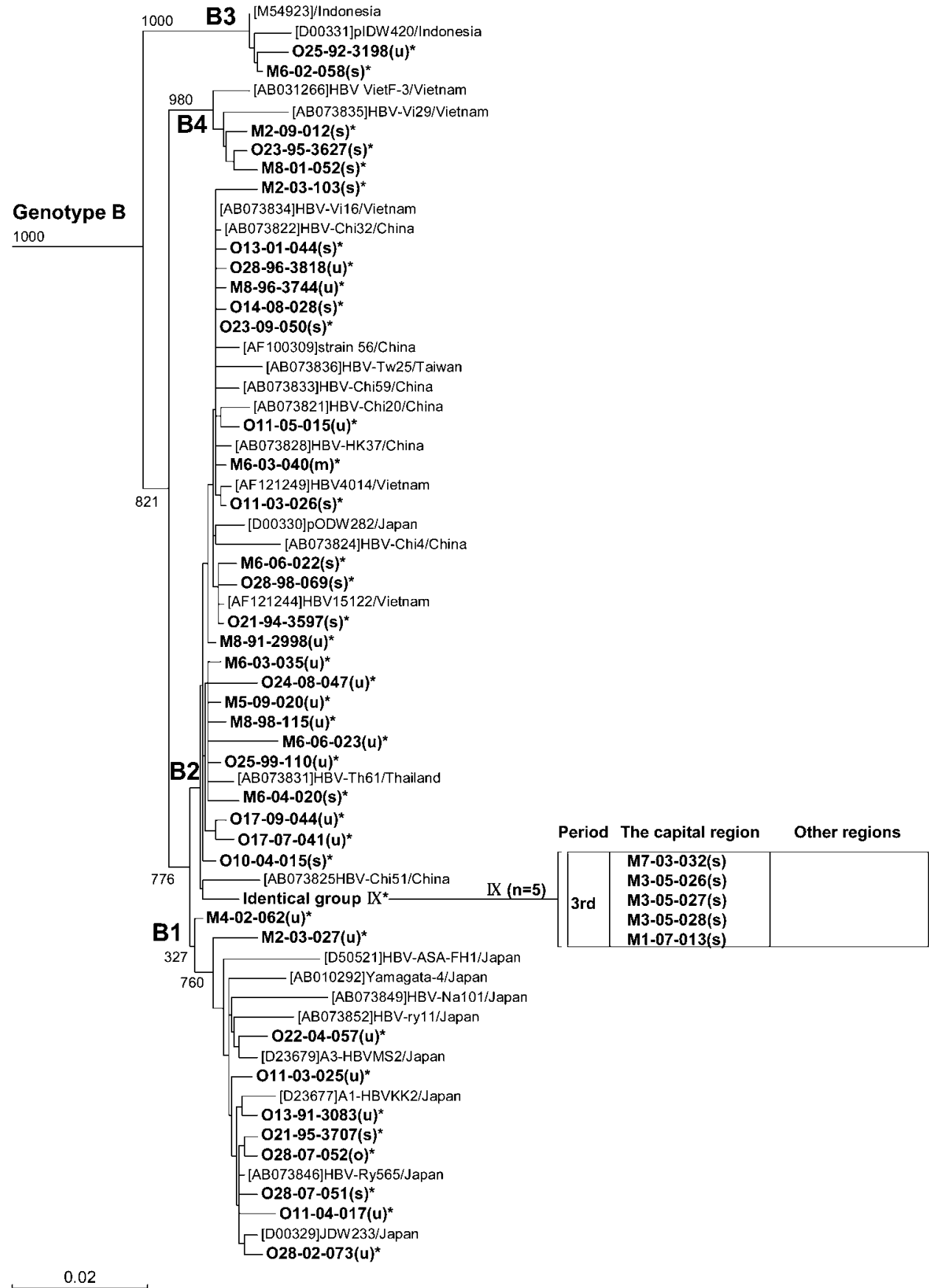

those with genotype C. Remarkably, all five patients with AHB who acquired chronic infection possessed HBV genotype A, either alone (four patients) or together with HBV genotype $\mathrm{G}$ (one). Increasing genotype A infections may have changed the genotype distribution in patients with $\mathrm{AHB}$ and those with chronic HBV infection. In Japanese patients with chronic hepatitis $\mathrm{B}$, the proportion of genotype $\mathrm{A}$ has doubled, from $1.7 \%$ in $1999-2000$ to $3.5 \%$ in $2005-2006 .^{37}$

The genotype was A in 29 of the 32 (91\%) homosexual men. Of the 29 homosexuals with genotype A, 10 gave consent to anti-HIV testing, and four of these (40\%) were found to be positive. Of the five patients who acquired chronic HBV infection, anti-HIV was tested in four (three with genotype $\mathrm{A}$ and one with genotypes A and G), and one with genotype A was found to be positive. There is a possibility that co-infecting HIV in this patient with genotype A may have promoted chronic
HBV infection; HIV is known to prolong and aggravate HBV infection by compromising immune responses. ${ }^{38}$

Patients with $\mathrm{FH}$ in this study were infected with either HBV genotype B (1/48 (2.1\%)) or C (8/359 (2.2\%)); no patients with genotype A developed FH. PreC and/or CP mutations were significantly less common in genotype A (1/109 (3.7\%)) than B $(6 / 39(15.4 \%))$ or C $(279 / 310(5.5 \%))$ infection. The single patient with genotype A who had PreC mutation was simultaneously infected with HBV genotype G. There is a possibility that the PreC mutation in this patient was from HBV genotype $\mathrm{G}^{26} \mathrm{FH}$ did not develop in any patients with genotype A, which may be attributable, at least in part, to the lack of PreC mutation in genotype $\mathrm{A}$ infections. ${ }^{39}$

Previous reports have shown that genotype $\mathrm{A}$ is common in patients with AHB in Metropolitan Tokyo, ${ }^{20} 2140$ as well as around Aichi located in the middle of Mainland Japan. ${ }^{22}$ 
Yotsuyanagi et $a^{23}$ reported that genotype $\mathrm{A}$ is more common in patients with $\mathrm{AHB}$ in the metropolitan region than in other regions. Sugauchi et $a l^{41}$ found that, in patients with $\mathrm{AHB}$, the proportion with genotype $\mathrm{A}$ has increased over time. The present study indicates that the number of patients with AHB in Japan would not have decreased. We found that the proportion of patients with genotype A infection is increasing in the 28 national hospitals in Japan $(6.0 \%$ in the 1 st period, $15.4 \%$ in the 2nd, and $39.4 \%$ in the 3rd (figure 2)), with the prevalence much higher in the capital than other regions (35.5\% vs $19.8 \%$ (table 2)).

In this study, there was a time lag in the increase in genotype A infection between the capital region and other regions of Japan (table 2). In the capital region, the prevalence of genotype A started to increase in the late 1990s, and kept increasing through the early 2000 s $(4.8 \%$ in the 1 st period, $29.3 \%$ in the 2nd, $50.0 \%$ in the $3 \mathrm{rd}$, and $56.3 \%$ in 2009). In other regions, by contrast, the frequency of genotype A did not change during the late $1990 \mathrm{~s}$, and increased significantly in the $2000 \mathrm{~s}(6.5 \%$ in the 1 st period, $8.5 \%$ in the $2 \mathrm{nd}, 33.1 \%$ in the $3 \mathrm{rd}$, and $34.4 \%$ in 2009). Thus infiltration of genotype A infection into other regions occurred 5-6years behind the epidemic in the capital region. This indicates that genotype $\mathrm{A}$ infection originated in the capital region and then spread to other areas of Japan.

Some genotypes are classified into several subgenotypes, and they have distinct geographical distributions. ${ }^{42}$ Hence, subgenotypes are useful in tracing the route of HBV infection. By phylogenetic analysis (figures 3 and 4), 88.6\% of genotype A isolates had the European-American type (A2), and the remaining $11.4 \%$ possessed the Asian-African type (A1). Likewise, $76.7 \%$ of genotype B isolates had Asian types (B2-B4), and the remaining $23.3 \%$ possessed the type endemic to Japan (B1). Of the $157 \mathrm{HBV}$ isolates of genotype A or B, 147 (93.6\%) had subgenotypes foreign to Japan. They are thought to have been transmitted from foreign sex workers, and spread among certain populations who share particular sexual behaviours in Japan. ${ }^{41}$

Of note, some HBV isolates of distinct subgenotypes possessed an identical sequence in the preS1/S2/S gene. The isolates of subgenotype A2 were prominent in this regard, and more often had the same sequence than those of other subgenotypes, such as A1, B1 and B2. The high prevalence of subgenotype A2 isolates with an identical sequence would not have been caused by cross-contamination. If cross-contamination had occurred, it would have affected isolates of all subgenotypes, and not influenced subgenotype A2 isolates preferentially. As many as $35 \%$ of subgenotype A2 isolates had an identical sequence, and those with the same sequence increased to $56.3 \%$ in the recent 2009 survey in Metropolitan Tokyo. Furthermore, some subgenotype A2 isolates in groups I, III and VII clustered locally within short periods, whereas others in groups II and VI were scattered widely over a long period of time. On the basis of these results, it is tempting to speculate that some subgenotype A2 strains would have been transmitted from person to person without undergoing mutations for many years.

In summary, the present study indicates the following. (1) AHB in the 28 national hospitals in Japan has not decreased, because genotype A infections are increasing. (2) Genotype A infections started to increase in the capital region, and then spread to local areas 5-6 years later. (3) Approximately $90 \%$ of genotype $\mathrm{A}$ in patients with $\mathrm{AHB}$ is subgenotype A2. (4) Subgenotype A2 strains with an identical sequence are spreading among younger generations with high sexual activity. (5) On the basis of the results obtained, AHB in Japan is not decreasing, because $\mathrm{HBV}$ of subgenotype $\mathrm{A} 2$ is prevailing in particular subpopulations at high risk. Finally, in order to prevent further increases in AHB in Japan, universal vaccination of young people deserves consideration.

\section{Author affiliations}

${ }^{1}$ Clinical Research Center, NHO Nagasaki Medical Center, Nagasaki, Japan ${ }^{2}$ Department of Hepatology, Nagasaki University Graduate School of Biomedical Sciences, Nagasaki, Japan

${ }^{3}$ National Center for Global Health and Medicine, Tokyo, Japan

${ }^{4} \mathrm{NHO}$ Kyushu Medical Center, Fukuoka, Japan

${ }^{5} \mathrm{NHO}$ Osaka National Hospital, Osaka, Japan

${ }^{6} \mathrm{NHO}$ Yokohama Medical Center, Kanagawa, Japan

${ }^{7} \mathrm{NHO}$ Sagamihara Hospital, Kanagawa, Japan

${ }^{8} \mathrm{NHO}$ Oita Medical Center, Oita, Japan

${ }^{9} \mathrm{NHO}$ Nagoya Medical Center, Aichi, Japan

${ }^{10} \mathrm{NHO}$ Osaka-Minami Medical Center, Osaka, Japan

${ }^{11} \mathrm{NHO}$ Kokura Medical Center, Fukuoka, Japan

${ }^{12} \mathrm{NHO}$ Sendai Medical Center, Miyagi, Japan

${ }^{13} \mathrm{NHO}$ Kyoto Medical Center, Kyoto, Japan

${ }^{14} \mathrm{NHO}$ Tokyo Medical Center, Tokyo, Japan

${ }^{15} \mathrm{NHO}$ Kure Medical Center and Chugoku Cancer Center, Hiroshima, Japan

${ }^{16} \mathrm{NHO}$ Kanazawa Medical Center, Ishikawa, Japan

${ }^{17} \mathrm{NHO}$ National Disaster Medical Center, Tokyo, Japan

${ }^{18}$ Miyakawa Memorial Research Foundation, Tokyo, Japan

Acknowledgements We thank participating physicians (MD) in the JNHAHSG for collaborating, with data and sample collection: Hideo Nishimura, Asahikawa Medical Center; Yukio Ohhara, Hokkaido Medical Center; Masakazu Kobayashi, and the late Takeshi Sodeyama, Matsumoto Medical Center; Fujio Makita, Nishigunma Hospital; Takeo Saoshiro, and Akira Saito, Nishisaitama-Chuo Hospital; Michiyasu Yagura, and the late Hideharu Harada, Tokyo Hospital; Keiichi Hirata, National Disaster Medical Center; Yoko Nakamura, Sagamihara Hospital; the late Hideo Morimoto, Kanazawa Medical Center; Noboru Hirashima, Higashi Nagoya National Hospital; Michio Kato, Osaka National Hospital; Tetsuo Yamamoto, Yonago Medical Center; Haruhiro Yamashita, Okayama Medical Center; Hirotaka Kouno and Eiichi Takezaki, Kure Medical Center and Chugoku Cancer Center; Toru Hayashi, and Shuji Oda, Zentsuji Hospital; Akihide Masumoto, Kokura Medical Center; Hironori Sakai, Beppu Medical Center; Koichi Honda, Oita Medical Center; Kazuhiro Sugi, Kumamoto Medical Center; Michiaki Koga, and Hiroaki Kawasoe, Ureshino Medical Center; Michitami Yano, and Manabu Daikoku, Nagasaki Medical Center. All the hospitals belong to National Hospital Organization (NHO). We appreciate the excellent technical assistance of Ms Rumiko Hamada, Ms Yuka Jiuchi, Ms Mika Fukuda and Ms Rumiko Nakao. Finally, we express our deep gratitude to the late Dr Koji Yano (Nagasaki Medical Center) for his contribution to the design and coordination of this study, and we dedicate this article to him.

Funding This study was supported by a grant-in-aid from the National Hospital Organization (grant number $\mathrm{H} 21-\mathrm{NHO}($ Kan)-03).

Competing interests None.

Ethics approval Approved by the ethics committee of each institution.

Contributors YT, HY and HI designed data collection tools, monitored data collection for the whole study, wrote the statistical analysis plan, cleaned and analysed the data YT, HY and YM drafted and revised the paper. HY, NM, MN, EM, TK, YW, TM, MS TH, TS, YM, TK, MT, HK, HO, SH and SA collaborated in data and sample collection.

Provenance and peer review Not commissioned; externally peer reviewed.

\section{REFERENCES}

1. Arauz-Ruiz P, Norder $\mathrm{H}$, Robertson BH, et al. Genotype $\mathrm{H}$ : a new Amerindian genotype of hepatitis B virus revealed in Central America. J Gen Virol 2002;83:2059-73.

2. Norder H, Courouce AM, Magnius LO. Complete genomes, phylogenetic relatedness, and structural proteins of six strains of the hepatitis B virus, four of which represent two new genotypes. Virology 1994;198:489-503.

3. Okamoto H, Tsuda F, Sakugawa H, et al. Typing hepatitis B virus by homology in nucleotide sequence: comparison of surface antigen subtypes. J Gen Virol 1988;69:2575-83.

4. Olinger CM, Jutavijittum P, Hubschen JM, et al. Possible new hepatitis B virus genotype, southeast Asia. Emerg Infect Dis 2008;14:1777-80.

5. Stuyver L, De Gendt S, Van Geyt C, et al. A new genotype of hepatitis B virus: complete genome and phylogenetic relatedness. J Gen Virol 2000;81:67-74.

6. Tran TT, Trinh TN, Abe K. New complex recombinant genotype of hepatitis B virus identified in Vietnam. J Virol 2008;82:5657-63.

7. Tatematsu K, Tanaka $Y$, Kurbanov F, et al. A genetic variant of hepatitis $B$ virus divergent from known human and ape genotypes isolated from a Japanese patient and provisionally assigned to new genotype J. J Virol 2009;83:10538-47. 
8. Chu CJ, Lok AS. Clinical significance of hepatitis B virus genotypes. Hepatology 2002;35:1274-6.

9. Kao JH. Hepatitis B viral genotypes: clinical relevance and molecular characteristics. J Gastroenterol Hepatol 2002;17:643-50.

10. Miyakawa Y, Mizokami M. Classifying hepatitis B virus genotypes. Intervirology 2003:46:329-38.

11. Kimbi GC, Kramvis A, Kew MC. Distinctive sequence characteristics of subgenotype A1 isolates of hepatitis B virus from South Africa. J Gen Virol 2004;85:1211-20.

12. Kramvis A, Weitzmann L, Owiredu WK, et al. Analysis of the complete genome of subgroup A' hepatitis B virus isolates from South Africa. J Gen Virol 2002;83:835-9.

13. Sugauchi F, Kumada $H$, Acharya $S A$, et al. Epidemiological and sequence differences between two subtypes ( $A e$ and $A a$ ) of hepatitis $B$ virus genotype $A$. $J$ Gen Virol 2004;85:811-20.

14. Sugauchi F, Kumada H, Sakugawa $H$, et al. Two subtypes of genotype B (Ba and Bj) of hepatitis B virus in Japan. Clin Infect Dis 2004;38:1222-8.

15. Sugauchi $\mathbf{F}$, Orito $E$, Ichida $T$, et al. Epidemiologic and virologic characteristics of hepatitis $B$ virus genotype $B$ having the recombination with genotype $C$.

Gastroenterology 2003;124:925-32.

16. Tanaka Y, Orito E, Yuen MF, et al. Two subtypes (subgenotypes) of hepatitis B virus genotype C: a novel subtyping assay based on restriction fragment length polymorphism. Hepatol Res 2005;33:216-24.

17. Akuta N, Suzuki F, Kobayashi M, et al. The influence of hepatitis B virus genotype on the development of lamivudine resistance during long-term treatment. J Hepatol 2003;38:315-21.

18. Tanaka Y, Hasegawa I, Kato T, et al. A case-control study for differences among hepatitis $B$ virus infections of genotypes $A$ (subtypes $A a$ and $A e$ ) and D. Hepatology 2004:40:747-55

19. Orito $\mathbf{E}$, Ichida $T$, Sakugawa $H$, et al. Geographic distribution of hepatitis B virus (HBV) genotype in patients with chronic HBV infection in Japan. Hepatology 2001;34:590-4.

20. Kobayashi $\mathbf{M}$, Arase $Y$, lkeda K, et al. Viral genotypes and response to interferon in patients with acute prolonged hepatitis $B$ virus infection of adulthood in Japan. J Med Virol 2002;68:522-8.

21. Ogawa $\mathbf{M}$, Hasegawa $K$, Naritomi $T$, et al. Clinical features and viral sequences of various genotypes of hepatitis $B$ virus compared among patients with acute hepatitis B. Hepatol Res 2002;23:167-77.

22. Takeda Y, Katano Y, Hayashi K, et al. Difference of HBV genotype distribution between acute hepatitis and chronic hepatitis in Japan. Infection 2006;34:201-7.

23. Yotsuyanagi H, Okuse C, Yasuda $\mathrm{K}$, et al. Distinct geographic distributions of hepatitis B virus genotypes in patients with acute infection in Japan. $J$ Med Virol 2005;77:39-46.

24. Kato H, Orito E, Sugauchi F, et al. Frequent coinfection with hepatitis B virus strains of distinct genotypes detected by hybridization with type-specific probes immobilized on a solid-phase support. J Virol Methods 2003;110:29-35.

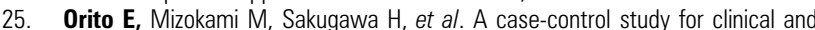
molecular biological differences between hepatitis $B$ viruses of genotypes $B$ and $C$. Japan HBV Genotype Research Group. Hepatology 2001;33:218-23.
26. Kato $\mathbf{H}$, Orito $\mathrm{E}$, Gish $\mathrm{RG}$, et al. Hepatitis $\mathrm{B}$ e antigen in sera from individuals infected with hepatitis B virus of genotype G. Hepatology 2002;35:922-9.

27. Sugiyama M, Tanaka Y, Sakamoto T, et al. Early dynamics of hepatitis B virus in chimeric mice carrying human hepatocytes monoinfected or coinfected with genotype G. Hepatology 2007;45:929-37.

28. Koyama T, Matsuda I, Sato S, et al. Prevention of perinatal hepatitis B virus transmission by combined passive-active immunoprophylaxis in Iwate, Japan (1981-1992) and epidemiological evidence for its efficacy. Hepatol Res 2003:26:287-92

29. Noto H, Terao T, Ryou S, et al. Combined passive and active immunoprophylaxis for preventing perinatal transmission of the hepatitis B virus carrier state in Shizuoka, Japan during 1980-1994. J Gastroenterol Hepatol 2003:18:943-9.

30. Tanaka J, Kumagai J, Katayama K, et al. Sex- and age-specific carriers of hepatitis $B$ and $C$ viruses in Japan estimated by the prevalence in the $3,485,648$ first-time blood donors during 1995-2000. Intervirology 2004;47:32-40.

31. Daniels D, Grytdal S, Wasley A. Surveillance for acute viral hepatitis-United States, 2007. MMWR Surveill Summ 2009:58:1-27.

32. Goldstein ST, Alter MJ, Williams IT, et al. Incidence and risk factors for acute hepatitis B in the United States, 1982-1998: implications for vaccination programs. $J$ Infect Dis 2002;185:713-19.

33. Sherlock S. The natural history of hepatitis B. Postgrad Med J 1987;63 Suppl 2:7-11.

34. Mayerat C, Mantegani A, Frei PC. Does hepatitis B virus (HBV) genotype influence the clinical outcome of HBV infection? J Viral Hepat 1999;6:299-304.

35. Hayashi K, Katano $Y$, Takeda $Y$, et al. Association of hepatitis B virus subgenotypes and basal core promoter/precore region variants with the clinical features of patients with acute hepatitis. J Gastroenterol 2008;43:558-64.

36. Ozasa A, Tanaka $Y$, Orito $E$, et al. Influence of genotypes and precore mutations on fulminant or chronic outcome of acute hepatitis B virus infection. Hepatology 2006;44:326-34

37. Matsuura K, Tanaka $Y$, Hige $S$, et al. Distribution of hepatitis B virus genotypes among patients with chronic infection in Japan shifting toward an increase of genotype A. J Clin Microbiol 2009;47:1476-83.

38. Bodsworth NJ, Cooper DA, Donovan B. The influence of human immunodeficiency virus type 1 infection on the development of the hepatitis B virus carrier state. J Infect Dis 1991;163:1138-40.

39. Li JS, Tong SP, Wen YM, et al. Hepatitis B virus genotype A rarely circulates as an $\mathrm{HBe}-$ minus mutant: possible contribution of a single nucleotide in the precore region. J Virol 1993:67:5402-10.

40. Suzuki Y, Kobayashi M, lkeda K, et al. Persistence of acute infection with hepatitis B virus genotype A and treatment in Japan. J Med Virol 2005;76:33-9.

41. Sugauchi F, Orito E, Ohno T, et al. Spatial and chronological differences in hepatitis $B$ virus genotypes from patients with acute hepatitis B in Japan. Hepatol Res 2006;36:107-14

42. Norder H, Courouce AM, Coursaget $\mathrm{P}$, et al. Genetic diversity of hepatitis B virus strains derived worldwide: genotypes, subgenotypes, and HBsAg subtypes. Intervirology 2004:47:289-309. 